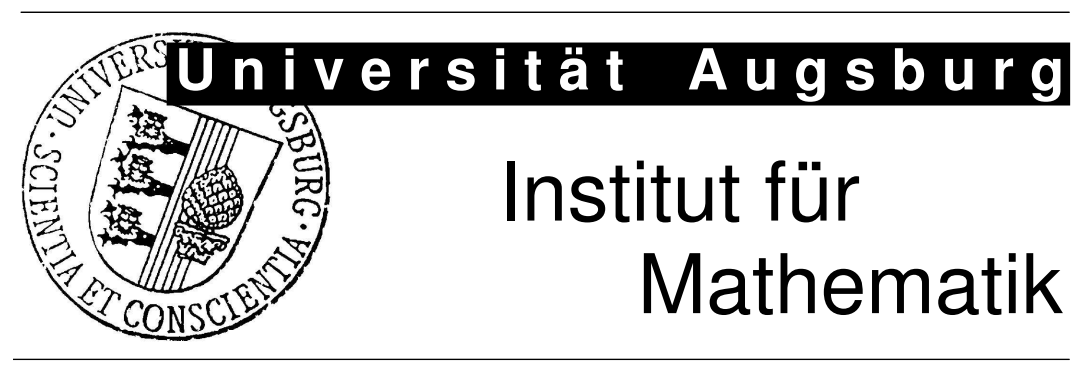

Carsten Carstensen, Ronald H.W. Hoppe

Unified Framework for an a Posteriori Error Analysis of

Non-Standard Finite Element Approximations of H(curl)-Elliptic Problems 


\section{Impressum:}

Herausgeber:

Institut für Mathematik

Universität Augsburg

86135 Augsburg

http: //www . math . uni-augsburg.de/forschung/preprint/

\section{ViSdP:}

Ronald H.W. Hoppe

Institut für Mathematik

Universität Augsburg

86135 Augsburg

Preprint: Sämtliche Rechte verbleiben den Autoren (c) 2009 


\title{
Unified Framework for an A Posteriori Error Analysis of Non-Standard Finite Element Approximations of $\mathbf{H}(\mathrm{curl})$ - Elliptic Problems
}

\author{
C. CARSTENSEN* and R. H. W. HOPPE ${ }^{\dagger+}$
}

22nd April 2009

\begin{abstract}
A unified framework for a residual-based a posteriori error analysis of standard conforming finite element methods as well as non-standard techniques such as nonconforming and mixed methods has been developed in [20]-[24]. This paper provides such a framework for an a posteriori error control of nonconforming finite element discretizations of $H$ (curl)-elliptic problems as they arise from low-frequency electromagnetics. These nonconforming approximations include the interior penalty discontinuous Galerkin (IPDG) approach considered in [33,34], and mortar edge element approximations studied in [10], [28]-[31], [41,48].
\end{abstract}

Keywords: a posteriori error analysis, unified framework, non-standard finite element methods, $H($ curl $)$-elliptic problems

\section{Dedicated to the Sixtieth Anniversary of Rolf Rannacher}

\section{INTRODUCTION}

The a posteriori error control and the design of adaptive mesh-refining algorithms is key to the actual scientific computing with any standard or nonstandard finite element method. The unifying theory of a posteriori error analysis [20]-[24] illustrates that all finite element methods allow for some a posteriori error control in energy norms for the Laplace, the Stokes, or the Lamé equations. This paper concerns the particular case of an $\mathbf{H}($ curl)-elliptic problem

$$
\operatorname{curl} \mu^{-1} \operatorname{curl} \mathbf{u}+\sigma \mathbf{u}=\mathbf{f}
$$

in a bounded polyhedral domain $\Omega \subset \mathbb{R}^{3}$ as it arises from a semi-discretization in time of the eddy current equations [35]. The idea is to rewrite the second-order PDE

\footnotetext{
* Dept. of Math., Humboldt-Universität zu Berlin, D-10099 Berlin, Germany

$\dagger$ Dept. of Math., University of Houston, Houston TX 77204-3008, U.S.A.

\$Inst. of Math., University of Augsburg, D-86159 Augsburg, Germany

The first author has been supported by the DFG Research Center MATHEON, Project C13. The second author acknowledges support by the NSF under Grants No. DMS-0707602, DMS-0810156, and DMS-0811153
} 
as a system of two first-order PDEs in weak form

$$
\mathscr{A}(\mathbf{u}, \mathbf{p})=\ell_{1}+\ell_{2} \text {. }
$$

Here, the operator $\mathscr{A}$ is given by

$$
(\mathscr{A}(\mathbf{u}, \mathbf{p}))(\mathbf{v}, \mathbf{q}):=\mathbf{a}(\mathbf{p}, \mathbf{q})-\mathbf{b}(\mathbf{u}, \mathbf{q})+\mathbf{b}(\mathbf{v}, \mathbf{p})+\mathbf{c}(\mathbf{u}, \mathbf{v})
$$

in terms of bilinear forms $\mathbf{a}, \mathbf{b}, \mathbf{c}$ and the linear functionals $\ell_{1}, \ell_{2}$ associated with the data of the problem (see Section 3 for details).

We prove in Proposition 3.1 that $\mathscr{A}$ is linear, bounded and bijective with bounded inverse. Therefore, the natural norms of any error is equivalent to the respective dual norms of the residuals.

Given some approximations $\tilde{\mathbf{u}}_{h}$ of $\mathbf{u}$ and $\tilde{\mathbf{p}}_{h}$ of $\mathbf{p}$, in the general analysis of residuals

$$
\begin{aligned}
& \operatorname{Res}_{1}(\mathbf{q}):=\ell_{1}(\mathbf{q})-\mathbf{a}\left(\tilde{\mathbf{p}}_{h}, \mathbf{q}\right)+\mathbf{b}\left(\tilde{\mathbf{u}}_{h}, \mathbf{q}\right), \\
& \operatorname{Res}_{2}(\mathbf{v}):=\ell_{2}(\mathbf{v})-\mathbf{b}\left(\mathbf{v}, \tilde{\mathbf{p}}_{h}\right)-\mathbf{c}\left(\tilde{\mathbf{u}}_{h}, \mathbf{v}\right)
\end{aligned}
$$

we rediscover the error estimators of $[7,8,32,43]$ for the curl-conforming edge elements of Nédélec's first family and those of [34] for an interior penalty discontinuous Galerkin method. In comparison with [34], the general framework even results in sharper estimates. In particular, with regard to the existing estimates with mesh-depending norms on the jumps, it is an innovative new feature of this paper (and of [21]) that those terms are obtained as known upper bounds while the consistency errors are actually smaller.

The remaining parts of this paper are organized as follows. Section 2 is devoted to the Sobolev spaces $\mathbf{H}(\operatorname{curl} ; \Omega)$ and $\mathbf{H}(\operatorname{div} ; \Omega)$ and various trace spaces thereof. The unified framework in Section 3 provides the details for the aforementioned operator $\mathscr{A}$ and the associated errors and residuals. Sections 4 and 5 recast the interior penalty discontinuous Galerkin method and the mortar edge element method in the above format and provide a new proof of the estimates in [34] and [31].

\section{H(CURL; $\Omega), H(D I V ; \Omega)$, AND THEIR TRACES}

Let $\Omega \subset \mathbb{R}^{3}$ be a simply connected polyhedral domain with boundary $\Gamma=\partial \Omega$ which can be split into $J$ open faces $\Gamma_{1}, \ldots, \Gamma_{J}$ with $\Gamma=\cup_{j=1}^{J} \bar{\Gamma}_{j}$. We denote by $\mathscr{D}(\Omega)$ the space of all infinitely often differentiable functions with compact support in $\Omega$ and by $\mathscr{D}^{\prime}(\Omega)$ its dual space referring to $<\cdot, \cdot>$ as the dual pairing between $\mathscr{D}^{\prime}(\Omega)$ and $\mathscr{D}(\Omega)$. We further adopt standard notation from Lebesgue and Sobolev space theory. We refer to $\mathbf{H}(\mathbf{c u r l} ; \Omega)$ as the linear space

$$
\mathbf{H}(\operatorname{curl} ; \Omega):=\left\{\mathbf{u} \in \mathbf{L}^{2}(\Omega) \mid \operatorname{curl} \mathbf{u} \in \mathbf{L}^{\mathbf{2}}(\Omega)\right\},
$$

which is a Hilbert space with respect to the inner product

$$
(\mathbf{u}, \mathbf{v})_{\operatorname{curl}, \Omega}:=(\mathbf{u}, \mathbf{v})_{0, \Omega}+(\operatorname{curl} \mathbf{u}, \operatorname{curl} \mathbf{v})_{0, \Omega} \quad \text { for all } \mathbf{u}, \mathbf{v} \in H(\operatorname{curl} ; \Omega)
$$


and associated norm $\|\cdot\|_{\text {curl }, \Omega}$. We further refer to $\mathbf{H}\left(\operatorname{curl}^{\mathbf{0}} ; \Omega\right)$ as the subspace of irrotational vector fields

$$
\mathbf{H}\left(\operatorname{curl}^{\mathbf{0}} ; \Omega\right)=\{\mathbf{u} \in \mathbf{H}(\operatorname{curl} ; \Omega) \mid \operatorname{curl} \mathbf{u}=\mathbf{0}\},
$$

which admits the characterization $\mathbf{H}\left(\operatorname{curl}^{\mathbf{0}} ; \Omega\right)=\operatorname{grad} H^{1}(\Omega)$. Its orthogonal complement

$$
\mathbf{H}^{\perp}(\operatorname{curl} ; \Omega)=\left\{\mathbf{u} \in \mathbf{H}(\operatorname{curl} ; \Omega) \mid\left(\mathbf{u}, \mathbf{u}^{\mathbf{0}}\right)_{0, \Omega}=0, \mathbf{u}^{\mathbf{0}} \in \mathbf{H}\left(\operatorname{curl}^{\mathbf{0}} ; \Omega\right)\right\}
$$

can be interpreted as the subspace of weakly solenoidal vector fields. The Hilbert space $\mathbf{H}(\mathbf{c u r l} ; \Omega)$ admits the following Helmholtz decomposition

$$
\mathbf{H}(\operatorname{curl} ; \Omega)=\mathbf{H}\left(\operatorname{curl}^{\mathbf{0}} ; \Omega\right) \oplus \mathbf{H}^{\perp}(\operatorname{curl} ; \Omega) .
$$

Likewise, the space $\mathbf{H}(\operatorname{div} ; \Omega)$ is defined by

$$
\mathbf{H}(\operatorname{div} ; \Omega):=\left\{\mathbf{q} \in \mathbf{L}^{2}(\Omega) \mid \operatorname{div} \mathbf{q} \in L^{2}(\Omega)\right\}
$$

which is a Hilbert space with respect to the inner product

$$
(\mathbf{u}, \mathbf{v})_{\operatorname{div}, \Omega}:=(\mathbf{u}, \mathbf{v})_{0, \Omega}+(\operatorname{div} \mathbf{u}, \operatorname{div} \mathbf{v})_{0, \Omega} \quad \text { for all } \mathbf{u}, \mathbf{v} \in H(\operatorname{div} ; \Omega)
$$

and associated norm $\|\cdot\|_{d i v, \Omega}$. For vector fields $\mathbf{u} \in \mathscr{D}(\bar{\Omega})^{3}:=\left\{\left.\varphi\right|_{\Omega} \mid \varphi \in \mathscr{D}\left(\mathbb{R}^{3}\right)\right\}$, the normal component trace reads

$$
\left.\eta_{\mathbf{n}}(\mathbf{u})\right|_{\Gamma_{j}}:=\left.\mathbf{n}_{j} \cdot \mathbf{u}\right|_{\Gamma_{j}} \text { for } j=1, \ldots, J
$$

with the exterior unit normal vector $\mathbf{n}_{j}$ on $\Gamma_{j}$. The normal component trace mapping can be extended by continuity to a surjective, continuous linear mapping (cf. [26]; Thm. 2.2)

$$
\eta_{\mathbf{n}}: \mathbf{H}(\operatorname{div} ; \Omega) \rightarrow \mathbf{H}^{-1 / 2}(\Gamma)
$$

We define $\mathbf{H}_{0}(\operatorname{div} ; \Omega)$ as the subspace of vector fields with vanishing normal components on $\Gamma$

$$
\mathbf{H}_{0}(\operatorname{div} ; \Omega):=\left\{\mathbf{u} \in \mathbf{H}(\operatorname{div} ; \Omega) \mid \eta_{\mathbf{n}}(\mathbf{u})=0\right\} .
$$

In order to study the traces of vector fields $\mathbf{q} \in H(\operatorname{curl} ; \Omega)$, following $[16,17,18]$, we introduce the spaces

$$
\begin{aligned}
\mathbf{L}_{\mathbf{t}}^{2}(\Gamma) & :=\left\{\mathbf{u} \in \mathbf{L}^{2}(\Omega) \mid \eta_{\mathbf{n}}(\mathbf{u})=0\right\} \\
\mathbf{H}_{-}^{1 / 2}(\Gamma) & :=\left\{\mathbf{u} \in \mathbf{L}_{\mathbf{t}}^{2}(\Gamma)|\mathbf{u}|_{\Gamma_{j}} \in \mathbf{H}^{1 / 2}\left(\Gamma_{j}\right) \text { for all } j=1, \ldots, J\right\} .
\end{aligned}
$$


For $\Gamma_{j}, \Gamma_{k} \subset \Gamma$ with $j \neq k$ and $E_{j k}:=\bar{\Gamma}_{j} \cap \bar{\Gamma}_{k} \in \mathscr{E}_{h}$, the set of edges, we denote by $\mathbf{t}_{j}$ and $\mathbf{t}_{k}$ the tangential unit vectors along $\Gamma_{j}$ and $\Gamma_{k}$ and by $\mathbf{t}_{j k}$ the unit vector parallel to $E_{j k}$ such that $\Gamma_{j}$ is spanned by $\mathbf{t}_{j}, \mathbf{t}_{j k}$ and $\Gamma_{k}$ by $\mathbf{t}_{k}, \mathbf{t}_{j k}$. Let

$$
\mathscr{I}_{k}:=\left\{j \in\{1, \ldots, N\} \mid \bar{\Gamma}_{j} \cap \bar{\Gamma}_{k}=E_{j k} \in \mathscr{E}_{h}\right\}
$$

and define

$$
\begin{aligned}
& \mathbf{H}_{\|}^{1 / 2}(\Gamma):=\left\{\mathbf{u} \in \mathbf{H}_{-}^{1 / 2}(\Gamma)\left|\left(\mathbf{t}_{j k} \cdot \mathbf{u}_{j}\right)\right|_{E_{j k}}=\left.\left(\mathbf{t}_{j k} \cdot \mathbf{u}_{k}\right)\right|_{E_{j k}} \text { for } k=1, \ldots, N \text { and } j \in \mathscr{I}_{k}\right\}, \\
& \mathbf{H}_{\perp}^{1 / 2}(\Gamma):=\left\{\mathbf{u} \in \mathbf{H}_{-}^{1 / 2}(\Gamma)\left|\left(\mathbf{t}_{j} \cdot \mathbf{u}_{j}\right)\right|_{E_{j k}}=\left(\mathbf{t}_{k} \cdot \mathbf{u}_{k}\right)_{E_{j k}} \text { for } k=1, \ldots, N \text { and } j \in \mathscr{I}_{k}\right\} .
\end{aligned}
$$

We refer to $\mathbf{H}_{\|}^{-1 / 2}(\Gamma)$ and $\mathbf{H}_{\perp}^{-1 / 2}(\Gamma)$ as the dual spaces of $\mathbf{H}_{\|}^{1 / 2}(\Gamma)$ and $\mathbf{H}_{\perp}^{1 / 2}(\Gamma)$ with $\mathbf{L}_{\mathbf{t}}^{2}(\Gamma)$ as the pivot space. For $\mathbf{u} \in \mathscr{D}(\bar{\Omega})^{3}$ we further define the tangential trace mapping

$$
\left.\gamma_{\mathbf{t}}\right|_{\Gamma_{j}}:=\left.\mathbf{u} \wedge \mathbf{n}_{j}\right|_{\Gamma_{j}} \text { for }=1, \ldots, n
$$

and the tangential components trace

$$
\left.\pi_{\mathbf{t}}\right|_{\Gamma_{j}}:=\left.\mathbf{n}_{j} \wedge\left(\mathbf{u} \wedge \mathbf{n}_{j}\right)\right|_{\Gamma_{j}} \text { for }=1, \ldots, n .
$$

Moreover, for a smooth function $u \in \mathscr{D}(\bar{\Omega})$ we define the tangential gradient operator $\nabla_{\Gamma}=\left.\operatorname{grad}\right|_{\Gamma}$ as the tangential components trace of the gradient operator $\nabla$

$$
\left.\nabla_{\Gamma} u\right|_{\Gamma_{j}}:=\nabla_{\Gamma_{j}} u=\pi_{\mathbf{t}, j}(\nabla u)=\mathbf{n}_{j} \wedge\left(\nabla u \wedge \mathbf{n}_{j}\right) \text { for }=1, \ldots, n,
$$

which leads to a continuous linear mapping $\nabla_{\Gamma}: H^{3 / 2}(\Gamma) \rightarrow \mathbf{H}_{\|}^{1 / 2}(\Gamma)$. The tangential divergence operator

$$
\left.\operatorname{div}\right|_{\tau}: \mathbf{H}_{\|}^{-1 / 2}(\Gamma) \rightarrow H^{-3 / 2}(\Gamma)
$$

is defined, with the respective dual pairings $\langle\cdot, \cdot\rangle$, as the adjoint operator of $-\nabla_{\Gamma}$

$$
\left\langle\left.\operatorname{div}\right|_{\Gamma} \mathbf{u}, v\right\rangle=-\left\langle\mathbf{u}, \nabla_{\Gamma} v\right\rangle \quad \text { for all } v \in H^{3 / 2}(\Gamma) \text { and } \mathbf{u} \in \mathbf{H}_{\|}^{-1 / 2}(\Gamma) .
$$

Finally, for $u \in \mathscr{D}(\Omega)$ we define the tangential curl operator $\operatorname{curl} \mid{ }_{\tau}$ as the tangential trace of the gradient operator

$$
\left.\operatorname{curl}_{\tau} u\right|_{\Gamma_{j}}=\left.\operatorname{curl}\right|_{\Gamma_{j}} u=\gamma_{\mathbf{t}, j}(\nabla u)=\nabla u \wedge \mathbf{n}_{j} \text { for } j=1, \ldots, n .
$$

The vectorial tangential curl operator is a linear continuous mapping

$$
\operatorname{curl}_{\tau}: H^{3 / 2}(\Gamma) \rightarrow \mathbf{H}_{\perp}^{1 / 2}(\Gamma) .
$$


The scalar tangential curl operator

$$
\operatorname{curl}_{\tau}: \mathbf{H}_{\perp}^{-1 / 2}(\Gamma) \rightarrow H^{-3 / 2}(\Gamma)
$$

is defined as the adjoint of the vectorial tangential curl operator via curl $\left.\right|_{\tau}$, i.e.,

$$
<\left.\operatorname{curl}\right|_{\tau} \mathbf{u}, v>=<\mathbf{u},\left.\operatorname{curl}\right|_{\Gamma} v>\quad \text { for all } \quad v \in H^{3 / 2}(\Gamma) \text { and } \mathbf{u} \in \mathbf{H}_{\perp}^{-1 / 2}(\Gamma) .
$$

The range spaces of the tangential trace mapping $\gamma_{\mathrm{t}}$ and the tangential components trace mapping $\pi_{\mathrm{t}}$ on $H(\mathbf{c u r l} ; \Omega)$ can be characterized by means of the spaces

$$
\begin{aligned}
\mathbf{H}^{-1 / 2}\left(\left.\operatorname{div}\right|_{\Gamma}, \Gamma\right) & :=\left\{\lambda \in \mathbf{H}_{\|}^{-1 / 2}(\Gamma)|\operatorname{div}|_{\Gamma} \lambda \in H^{-1 / 2}(\Gamma)\right\}, \\
\mathbf{H}^{-1 / 2}\left(\left.\operatorname{curl}\right|_{\Gamma}, \Gamma\right) & :=\left\{\lambda \in \mathbf{H}_{\perp}^{-1 / 2}(\Gamma)|\operatorname{curl}|_{\Gamma} \lambda \in H^{-1 / 2}(\Gamma)\right\},
\end{aligned}
$$

which are dual to each other with respect to the pivot space $\mathbf{L}_{\mathbf{t}}^{2}(\Gamma)$. We refer to $\|\cdot\|_{-1 / 2, d i v_{\Gamma}, \Gamma}$ and $\|\cdot\|_{-1 / 2, \operatorname{cur}_{\Gamma}, \Gamma}$ as the respective norms and denote by $\langle\cdot, \cdot\rangle_{-1 / 2, \Gamma}$ the dual pairing (see, e.g., [18] for details).

It can be shown that the tangential trace mapping is a continuous linear mapping

$$
\gamma_{\mathbf{t}}: \mathbf{H}(\operatorname{curl} ; \Omega) \rightarrow \mathbf{H}^{-1 / 2}\left(\left.\operatorname{div}\right|_{\Gamma}, \Gamma\right),
$$

whereas the tangential components trace mapping is a continuous linear mapping

$$
\pi_{\mathbf{t}}: \mathbf{H}(\operatorname{curl} ; \Omega) \rightarrow \mathbf{H}^{-1 / 2}\left(\left.\operatorname{curl}\right|_{\Gamma}, \Gamma\right) .
$$

The previous results imply that the tangential divergence of the tangential trace and the scalar tangential curl of the tangential components trace coincide: For $\mathbf{u} \in$ $\mathbf{H}(\mathbf{c u r l} ; \Omega)$ it holds

$$
\left.\operatorname{div}\right|_{\Gamma}(\mathbf{u} \wedge \mathbf{n})=\left.\operatorname{curl}\right|_{\Gamma}(\mathbf{n} \wedge(\mathbf{u} \wedge \mathbf{n}))=\mathbf{n} \cdot \operatorname{curl} \mathbf{u} .
$$

We define $\mathbf{H}_{\mathbf{0}}(\mathbf{c u r l} ; \Omega)$ as the subspace of $\mathbf{H}(\mathbf{c u r l} ; \Omega)$ with vanishing tangential traces on $\Gamma$

$$
\mathbf{V}:=\mathbf{H}_{\mathbf{0}}(\operatorname{curl} ; \Omega):=\left\{\mathbf{u} \in \mathbf{H}(\operatorname{curl} ; \Omega) \mid \gamma_{\mathbf{t}}(\mathbf{u})=0\right\}
$$

\section{THE UNIFIED FRAMEWORK}

As a model problem, for given $\mathbf{f} \in \mathbf{H}(\operatorname{div} ; \Omega)$ and $\mu>0, \sigma>0$, we consider the following elliptic boundary-value problem (BVP)

$$
\begin{array}{rlrl}
\operatorname{curl} \mu^{-1} \operatorname{curl} \mathbf{u}+\sigma \mathbf{u} & =\mathbf{f} & & \text { in } \Omega, \\
\gamma_{\mathbf{t}}(\mathbf{u})=0 & & \text { on } \Gamma .
\end{array}
$$


This BVP can be interpreted as the stationary form of the 3D eddy currents equations with $\mu, \sigma$ being related to the magnetic permeability and electric conductivity, respectively, and $\mathbf{f}$ standing for a current density. The weak formulation of (3.1a)(3.1b) amounts to the computation of $\mathbf{u} \in \mathbf{H}_{\mathbf{0}}(\mathbf{c u r l} ; \Omega)$ such that

$$
\int_{\Omega}\left(\mu^{-1} \mathbf{u} \cdot \operatorname{curl} \mathbf{v}+\sigma \mathbf{u} \cdot \mathbf{v}\right) d x=\int_{\Omega} \mathbf{f} \cdot \mathbf{v} d x \text { for all } \mathbf{v} \in \mathbf{H}_{\mathbf{0}}(\operatorname{curl} ; \Omega) .
$$

With $\mathbf{p}:=\mu^{-1}$ curl $\mathbf{u} \in \mathbf{L}^{\mathbf{2}}(\Omega)$, (3.1a) can be recast as the first-order system

$$
\begin{aligned}
& \mu \mathbf{p}-\operatorname{curl} \mathbf{u}=0, \\
& \operatorname{curl} \mathbf{p}+\sigma \mathbf{u}=\mathbf{f} .
\end{aligned}
$$

The fundamental Hilbert spaces

$$
\mathbf{V}:=\mathbf{H}_{\mathbf{0}}(\operatorname{curl} ; \Omega) \quad \text { and } \quad \mathbf{Q}:=\mathbf{L}^{\mathbf{2}}(\Omega)
$$

allow for the definition of the bilinear forms

$$
\mathbf{a}(\cdot, \cdot): \mathbf{Q} \times \mathbf{Q} \rightarrow \mathbb{R}, \mathbf{b}(\cdot, \cdot): \mathbf{V} \times \mathbf{Q} \rightarrow \mathbb{R}, \text { and } \mathbf{c}(\cdot, \cdot): \mathbf{V} \times \mathbf{V} \rightarrow \mathbb{R}
$$

as well as functionals $\ell_{1} \in \mathbf{Q}^{*}$ and $\ell_{2} \in \mathbf{V}^{*}$ according to

$$
\begin{aligned}
\mathbf{a}(\mathbf{p}, \mathbf{q}) & :=\int_{\Omega} \mu \mathbf{p} \cdot \mathbf{q} d x \quad \text { for all } \mathbf{p}, \mathbf{q} \in \mathbf{Q}, \\
\mathbf{b}(\mathbf{u}, \mathbf{q}) & :=\int_{\Omega} \operatorname{curl}_{h} \mathbf{u} \cdot \mathbf{q} d x \quad \text { for all } \mathbf{u} \in \mathbf{V}, \mathbf{q} \in \mathbf{Q}, \\
\mathbf{c}(\mathbf{u}, \mathbf{v}) & :=\int_{\Omega} \sigma \mathbf{u} \cdot \mathbf{v} d x \quad \text { for all } \mathbf{u}, \mathbf{v} \in \mathbf{V}, \\
\ell_{1}(\mathbf{q}) & :=0 \text { for all } \mathbf{q} \in \mathbf{Q}, \\
\ell_{2}(\mathbf{v}) & :=\int_{\Omega} \mathbf{f} \cdot \mathbf{v} d x \quad \text { for all } \mathbf{v} \in \mathbf{V} .
\end{aligned}
$$

Here and throughout the paper, $\operatorname{curl}_{h}$ refers to the piecewise action of the curloperator used later for discrete vector-valued functions (note that $\operatorname{curl}_{h} \mathbf{u}=\operatorname{curl} \mathbf{u}$ for $\mathbf{u} \in \mathbf{V}$ ) and $\ell_{1} \in \mathbf{Q}^{*}$ has been formally introduced for later purposes as well. The weak formulation of (3.3a)-(3.3b) is to find $(\mathbf{u}, \mathbf{p}) \in \mathbf{V} \times \mathbf{Q}$ such that

$$
\begin{array}{ll}
\mathbf{a}(\mathbf{p}, \mathbf{q})-\mathbf{b}(\mathbf{u}, \mathbf{q})=\ell_{1}(\mathbf{q}) & \text { for all } \mathbf{q} \in \mathbf{Q}, \\
\mathbf{b}(\mathbf{v}, \mathbf{p})+\mathbf{c}(\mathbf{u}, \mathbf{v})=\ell_{2}(\mathbf{v}) & \text { for all } \mathbf{v} \in \mathbf{V} .
\end{array}
$$

The operator-theoretic framework involves the operator $\mathscr{A}:(\mathbf{V} \times \mathbf{Q}) \rightarrow(\mathbf{V} \times \mathbf{Q})^{*}$ defined, for all $(\mathbf{u}, \mathbf{p}),(\mathbf{v}, \mathbf{q}) \in \mathbf{V} \times \mathbf{Q}$, by

$$
(\mathscr{A}(\mathbf{u}, \mathbf{p}))(\mathbf{v}, \mathbf{q}):=\mathbf{a}(\mathbf{p}, \mathbf{q})-\mathbf{b}(\mathbf{u}, \mathbf{q})+\mathbf{b}(\mathbf{v}, \mathbf{p})+\mathbf{c}(\mathbf{u}, \mathbf{v}) .
$$


Then, the system (3.5a)-(3.5b) is recast in compact form as

$$
\mathscr{A}(\mathbf{u}, \mathbf{p})=\ell_{1}+\ell_{2} .
$$

Proposition 3.1. For positive $\mu, \sigma$, the operator $\mathscr{A}$ is a continuous, linear, and bijective and, hence, $\mathscr{A}$ has a bounded inverse.

Proof. The mapping properties are straightforward and the proof here focuses on the bijectivity which essentially follows from the inf-sup condition. In fact, given any $(\mathbf{u}, \mathbf{p}) \in \mathbf{V} \times \mathbf{Q}$ one calculates

$$
\begin{aligned}
(\mathscr{A}(\mathbf{u}, \mathbf{p}))\left(3 \mathbf{u}, 2 \mathbf{p}-\mu^{-1} \operatorname{curl}_{h} \mathbf{u}\right) & =\left(\mathscr{A}\left(3 \mathbf{u}, 2 \mathbf{p}+\mu^{-1} \operatorname{curl}_{h} \mathbf{u}\right)\right)(\mathbf{u}, \mathbf{p}) \\
& =2 \mu\|\mathbf{p}\|_{L^{2}(\Omega)}^{2}+3 \sigma\|\mathbf{u}\|_{L^{2}(\Omega)}^{2}+\mu^{-1}\left\|\operatorname{curl}_{h} \mathbf{u}\right\|_{L^{2}(\Omega)}^{2} .
\end{aligned}
$$

This implies the inf-sup condition and the remaining degeneracy condition which leads to bijectivity.

As an immediate consequence, given any $\ell_{1} \in \mathbf{Q}^{*}, \ell_{2} \in \mathbf{V}^{*}$, there exists a unique solution $(\mathbf{u}, \mathbf{p}) \in \mathbf{V} \times \mathbf{Q}$ of (3.7). Moreover, given any $\left(\tilde{\mathbf{u}}_{h}, \tilde{\mathbf{p}}_{h}\right) \in \mathbf{V} \times \mathbf{Q}$, it holds

$$
\left\|\left(\mathbf{u}-\tilde{\mathbf{u}}_{h}, \mathbf{p}-\tilde{\mathbf{p}}_{h}\right)\right\|_{\mathbf{V} \times \mathbf{Q}} \approx\left\|\operatorname{Res}_{\mathbf{1}}\right\|_{\mathbf{Q}^{*}}+\left\|\operatorname{Res}_{\mathbf{2}}\right\|_{\mathbf{V}^{*}}
$$

with residuals $\operatorname{Res}_{\mathbf{1}} \in \mathbf{Q}^{*}$ and $\operatorname{Res}_{\mathbf{2}} \in \mathbf{V}^{*}$,

$$
\begin{aligned}
& \operatorname{Res}_{1}(\mathbf{q}):=\ell_{1}(\mathbf{q})-\mathbf{a}\left(\tilde{\mathbf{p}}_{h}, \mathbf{q}\right)+\mathbf{b}\left(\tilde{\mathbf{u}}_{h}, \mathbf{q}\right) \quad \text { for all } \mathbf{q} \in \mathbf{Q}, \\
& \operatorname{Res}_{2}(\mathbf{v}):=\ell_{2}(\mathbf{v})-\mathbf{b}\left(\mathbf{v}, \tilde{\mathbf{p}}_{h}\right)-\mathbf{c}\left(\tilde{\mathbf{u}}_{h}, \mathbf{v}\right) \quad \text { for all } \mathbf{v} \in \mathbf{V} .
\end{aligned}
$$

The first residual $\operatorname{Res}_{\mathbf{1}}(\mathbf{q})$ equals the function $\tilde{\mathbf{p}}_{h}-\mu^{-1} \operatorname{curl}_{h} \tilde{\mathbf{u}}_{h}$ times the test function $\mathbf{q}$ in the scalar product of $\mathbf{L}^{2}(\Omega)$. The corresponding dual norm is therefore the $\mathbf{L}^{2}(\Omega)$ norm of $\tilde{\mathbf{p}}_{h}-\mu^{-1} \operatorname{curl}_{h} \tilde{\mathbf{u}}_{h}$, i.e.,

$$
\left\|\operatorname{Res}_{\mathbf{1}}\right\|_{\mathbf{Q}^{*}}=\left\|\tilde{\mathbf{p}}_{h}-\mu^{-1} \operatorname{curl}_{h} \tilde{\mathbf{u}}_{h}\right\|_{0, \Omega} .
$$

The analysis of the second residual $\mathbf{R e s}_{2}$ involves an integration by parts and some dual norm with test functions in $\mathbf{V}$. Therefore, the analysis of $\left\|\mathbf{R e s}_{\mathbf{2}}\right\| \mathbf{V}^{*}$ is more involved and requires additional properties from the weak form and the discrete solutions.

We assume $\mathscr{T}_{h}$ to be a regular simplicial triangulation with $\mathscr{E}_{h}(D)$ and $\mathscr{F}_{h}(D)$ denoting the sets of edges and faces of $\mathscr{T}_{h}$ in $D \subset \bar{\Omega}$. The curl-conforming edge elements of Nédélec's first family with respect to $T \in \mathscr{T}_{h}$ read

$$
\mathbf{N d}_{\mathbf{1}}(T):=\left\{\mathbf{v} \mid \exists \mathbf{a}, \mathbf{b} \in \mathbb{R}^{3}, \forall \mathbf{x} \in T, \mathbf{v}(\mathbf{x}):=\mathbf{a}+\mathbf{b} \wedge \mathbf{x}\right\}
$$

with degrees of freedom given by the zero-order moments of the tangential components along the edges $E \in \mathscr{E}_{h}(T)$ and

$$
\operatorname{Nd}_{\mathbf{1}}\left(\Omega ; \mathscr{T}_{h}\right):=\left\{\mathbf{v}_{h} \in \mathbf{V}\left|\forall T \in \mathscr{T}_{h}, \mathbf{v}_{h}\right|_{T} \in \mathbf{N d}_{\mathbf{1}}(T)\right\} .
$$


Under the condition

$$
\operatorname{Nd}_{\mathbf{1}}\left(\Omega ; \mathscr{T}_{h}\right) \subset \operatorname{Ker} \operatorname{Res}_{2},
$$

reliability holds for the explicit residual-based error estimator which, for each $T \in$ $\mathscr{T}_{h}$ and with tangential and normal jumps across interior faces $F \in \mathscr{F}_{h}(\Omega)$, reads

$$
\begin{aligned}
\eta_{T} & :=h_{T}\left\|\mathbf{f}-\sigma \tilde{\mathbf{u}}_{h}-\operatorname{curl}_{h} \tilde{\mathbf{p}}_{h}\right\|_{0, T}+h_{T}\left\|\operatorname{div}\left(\mathbf{f}-\sigma \tilde{\mathbf{u}}_{h}\right)\right\|_{0, T}, \\
\eta_{F} & :=h_{F}^{1 / 2}\left\|\left[\pi_{t}\left(\tilde{\mathbf{p}}_{h}\right)\right]\right\|_{0, F}+h_{F}^{1 / 2}\left\|\mathbf{n}_{F} \cdot\left[\sigma \tilde{\mathbf{u}}_{h}\right]\right\|_{0, F} .
\end{aligned}
$$

Proposition $3.2[32,43]$. Using the notation before and under the condition (3.11) it holds

$$
\left\|\operatorname{Res}_{\mathbf{2}}\right\|_{\mathbf{V}^{*}}^{2} \lesssim \eta^{2}:=\sum_{T \in \mathscr{T}_{h}} \eta_{T}^{2}+\sum_{F \in \mathscr{F}_{h}(\Omega)} \eta_{F}^{2} .
$$

Proof. Given any $\mathbf{v} \in \mathbf{V}$, Theorem 1 of [43] shows that there exist $\mathbf{v}_{h} \in \mathbf{N d}_{\mathbf{1}}\left(\Omega ; \mathscr{T}_{h}\right)$, $\varphi \in H_{0}^{1}(\Omega)$, and $\mathbf{z} \in H_{0}^{1}(\Omega)^{3}$ with

$$
\mathbf{v}-\mathbf{v}_{h}=\nabla \varphi+\mathbf{z}
$$

plus approximation and stability properties. The proof then follows that of Corollary 2 of [43] for

$$
\operatorname{Res}_{\mathbf{2}}(\mathbf{v})=\operatorname{Res}_{\mathbf{2}}\left(\mathbf{v}-\mathbf{v}_{h}\right)=\operatorname{Res}_{\mathbf{2}}(\nabla \varphi+\mathbf{z})
$$

and employs integration by parts followed by trace inequalities and approximation estimates of $\nabla \varphi$ and $\mathbf{z}$. Since the proof in [43] is quite explicit, details are dropped here.

The converse estimate holds up to data oscillations $[8,32]$.

\section{INTERIOR PENALTY DISCONTINUOUS GALERKIN METHODS}

Let $\mathscr{T}_{h}$ be a geometrically conforming, shape-regular simplicial triangulation of $\Omega$. The discrete spaces $\mathbf{V}_{h}$ and $\mathbf{Q}_{h}$ are chosen as elementwise polynomials of degree $\leqslant p$

$$
\mathbf{V}_{h}:=\Pi_{p}\left(\mathscr{T}_{h} ; \mathbb{R}^{3}\right) \quad \text { and } \quad \mathbf{Q}_{h}:=\Pi_{p}\left(\mathscr{T}_{h} ; \mathbb{R}^{3}\right) .
$$

For this choice and some penalty parameter $\alpha \geqslant \alpha_{\min }>0$, set

$$
\begin{aligned}
& \mathbf{J}_{\mathbf{1}}\left(\mathbf{v}_{h}, \mathbf{q}_{h}\right):=\sum_{F \in \mathscr{F}_{h}(\Omega)} \int_{F}\left\{\pi_{t}\left(\mathbf{q}_{h}\right)\right\} \cdot\left[\gamma_{t}\left(\mathbf{v}_{h}\right)\right] d s, \\
& \mathbf{J}_{2}\left(\mathbf{u}_{h}, \mathbf{v}_{h}\right):=\sum_{F \in \mathscr{F}_{h}(\Omega)} \int_{F}\left(\left\{\pi_{t}\left(\operatorname{curl} \mathbf{u}_{h}\right)\right\}-\alpha\left[\gamma_{t}\left(\mathbf{u}_{h}\right)\right]\right) \cdot\left(\left[\gamma_{t}\left(\mathbf{v}_{h}\right)\right]\right) d s .
\end{aligned}
$$


The first formulation of the Interior Penalty Discontinuous Galerkin Method reads: Find $\left(\mathbf{u}_{h}, \mathbf{p}_{h}\right) \in \mathbf{V}_{h} \times \mathbf{Q}_{h}$ such that

$$
\begin{aligned}
\mathbf{a}\left(\mathbf{p}_{h}, \mathbf{q}_{h}\right)-\mathbf{b}\left(\mathbf{u}_{h}, \mathbf{q}_{h}\right)=\ell_{1}\left(\mathbf{q}_{h}\right)+\mathbf{J}_{1}\left(\mathbf{u}_{h}, \mathbf{q}_{h}\right) & \text { for all } \mathbf{q}_{h} \in \mathbf{Q}_{h}, \\
\mathbf{b}\left(\mathbf{v}_{h}, \mathbf{p}_{h}\right)+\mathbf{c}\left(\mathbf{u}_{h}, \mathbf{v}_{h}\right)=\ell_{2}\left(\mathbf{v}_{h}\right)+\mathbf{J}_{2}\left(\mathbf{u}_{h}, \mathbf{v}_{h}\right) & \text { for all } \mathbf{v}_{h} \in \mathbf{V}_{h} .
\end{aligned}
$$

The second formulation in the primal variable reads: Find $\mathbf{u}_{h} \in \mathbf{V}_{h}$ such that, for all $\mathbf{v}_{h} \in \mathbf{V}_{h}$, it holds

$$
\begin{aligned}
& \mathbf{c}\left(\mathbf{u}_{h}, \mathbf{v}_{h}\right)+\sum_{T \in \mathscr{T}_{h}}\left(\mu^{-1} \operatorname{curl} \mathbf{u}_{h}, \operatorname{curl} \mathbf{v}_{h}\right)_{0, T} \\
& =\ell_{1}\left(\mu^{-1} \operatorname{curl}_{h}\right)+\ell_{2}\left(\mathbf{v}_{h}\right)+\mathbf{J}_{\mathbf{1}}\left(\mathbf{u}_{h}, \mu^{-1} \operatorname{curl} \mathbf{v}_{h}\right)+\mathbf{J}_{\mathbf{2}}\left(\mathbf{u}_{h}, \mathbf{v}_{h}\right) .
\end{aligned}
$$

Theorem 4.1. The formulations (4.1a)-(4.1b) and (4.2) are formally equivalent in the following sense. If $\left(\mathbf{u}_{h}, \mathbf{p}_{h}\right) \in \mathbf{V}_{h} \times \mathbf{Q}_{h}$ solves (4.1a)-(4.1b), then $\mathbf{u}_{h} \in \mathbf{V}_{h}$ solves (4.2). Conversely, if $\mathbf{u}_{h} \in \mathbf{V}_{h}$ solves (4.2), then there exists some $\mathbf{p}_{h} \in \mathbf{Q}_{h}$ such that $\left(\mathbf{u}_{h}, \mathbf{p}_{h}\right)$ solves $(4.1 \mathrm{a})-(4.1 \mathrm{~b})$.

Proof. Suppose that $\left(\mathbf{u}_{h}, \mathbf{p}_{h}\right) \in \mathbf{V}_{h} \times \mathbf{Q}_{h}$ solves (4.1a)-(4.1b). Since $\mu$ is constant on each element $T \in \mathscr{T}_{h}, \mathbf{q}_{h}:=\mu^{-1}$ curl $\mathbf{v}_{h}$ is a proper test function in (4.1a) for any $\mathbf{v}_{h} \in \mathbf{V}_{h}$. The resulting identity involves

$$
\mathbf{a}\left(\mathbf{p}_{h}, \mu^{-1} \operatorname{curl}_{h}\right)=\mathbf{b}\left(\mathbf{v}_{h}, \mathbf{p}_{h}\right) .
$$

This and (4.1b) imply (4.2).

Conversely, let $\mathbf{u}_{h} \in \mathbf{V}_{h}$ solve (4.2). Then, the expression

$$
\mathbf{b}\left(\mathbf{u}_{h}, \mathbf{q}_{h}\right)+\ell_{1}\left(\mathbf{q}_{h}\right)+\mathbf{J}_{\mathbf{1}}\left(\mathbf{u}_{h}, \mathbf{q}_{h}\right)
$$

is a linear and bounded functional as a function of $\mathbf{q}_{h} \in \mathbf{Q}_{h}$. Since $\mathbf{a}$ is a scalar product on $\mathbf{Q}_{h}$, there exists a unique Riesz representation $\mathbf{a}\left(\mathbf{p}_{h}, \cdot\right)$ of this linear functional. Then, $\left(\mathbf{u}_{h}, \mathbf{p}_{h}\right) \in \mathbf{V}_{h} \times \mathbf{Q}_{h}$ solves (4.1a). Again, $\mathbf{q}_{h}:=\mu^{-1}$ curl $\mathbf{v}_{h}$ is a proper test function in (4.1a). The resulting expression combined with (4.2) allows the proof of (4.1b).

Given the solution $\left(\mathbf{u}_{h}, \mathbf{p}_{h}\right) \in \mathbf{V}_{h} \times \mathbf{Q}_{h}$ of (4.1a)-(4.1b), consider the consistency error

$$
\xi:=\min _{\tilde{\mathbf{v}}_{h} \in \mathbf{V}}\left(\left\|\mathbf{u}_{h}-\tilde{\mathbf{v}}_{h}\right\|_{L^{2}(\Omega)}^{2}+\left\|\operatorname{curl}_{h} \mathbf{u}_{h}-\operatorname{curl} \tilde{\mathbf{v}}_{h}\right\|_{L^{2}(\Omega)}^{2}\right)^{1 / 2}
$$

and notice that the minimum is attained with a minimiser $\tilde{\mathbf{u}}_{h} \in \mathbf{V}$, i.e.,

$$
\xi^{2}=\left\|\mathbf{u}_{h}-\tilde{\mathbf{u}}_{h}\right\|_{L^{2}(\Omega)}^{2}+\left\|\operatorname{curl}_{h} \mathbf{u}_{h}-\operatorname{curl} \tilde{\mathbf{u}}_{h}\right\|_{L^{2}(\Omega)}^{2} .
$$

Since there exist computable upper bounds for $\xi$, it is not necessary to compute the minimiser $\tilde{\mathbf{u}}_{h} \in \mathbf{V}$ for error control. For instance, in Proposition 4.1 of [34], it is shown that

$$
\xi^{2} \lesssim \alpha \sum_{F \in \mathscr{F}_{h}(\Omega)} h_{F}^{-1}\left\|\left[\gamma_{t}\left(\mathbf{u}_{h}\right)\right]\right\|_{0, F}^{2}=: \bar{\xi}^{2}
$$


Since, the jumps are also error terms, e.g.,

$$
h_{F}^{-1}\left\|\left[\gamma_{t}\left(\mathbf{u}_{h}\right)\right]\right\|_{0, F}^{2}=h_{F}^{-1}\left\|\left[\gamma_{t}\left(\mathbf{u}-\mathbf{u}_{h}\right)\right]\right\|_{0, F}^{2},
$$

they are seen as a contribution to the DG error norm and, at the same time, are computable a posteriori and so arise in the upper bounds in [34] . However, in this paper, we consider those jump contributions $\bar{\xi}$ as one known upper bound of $\xi$ whose efficiency is less clear to us.

Given the aforementioned minimiser $\tilde{\mathbf{u}}_{h} \in \mathbf{V}$ in the definition of $\xi$, we let

$$
\tilde{\mathbf{p}}_{h}:=\mu^{-1} \operatorname{curl} \tilde{\mathbf{u}}_{h} \in \mathbf{Q} .
$$

Then, the unified approach leads to (3.8) with the residuals (3.9a)-(3.9b). Here,

$$
\operatorname{Res}_{\mathbf{1}}(\mathbf{q})=0 \quad \text { for all } \mathbf{q} \in \mathbf{Q}
$$

and, for all $\mathbf{v} \in \mathbf{V}$,

$$
\operatorname{Res}_{2}(\mathbf{v}):=\int_{\Omega}\left(\mathbf{f} \cdot \mathbf{v}-\mu^{-1} \operatorname{curl}_{h} \tilde{\mathbf{u}}_{h} \cdot \operatorname{curl} \mathbf{v}-\sigma \tilde{\mathbf{u}}_{h} \cdot \mathbf{v}\right) d x .
$$

Lemma 4.1. For any $\mathbf{v}_{h} \in \mathbf{N d}_{\mathbf{1}}\left(\Omega ; \mathscr{T}_{h}\right)$, it holds

$$
\operatorname{Res}_{\mathbf{2}}\left(\mathbf{v}_{h}\right)=\mathbf{c}\left(\mathbf{u}_{h}-\tilde{\mathbf{u}}_{h}, \mathbf{v}_{h}\right) .
$$

Proof. Since $\mathbf{v}_{h} \in \mathbf{N d}_{\mathbf{1}}\left(\Omega ; \mathscr{T}_{h}\right) \subset \Pi_{p}\left(\mathscr{T}_{h} ; \mathbb{R}^{3}\right)$ is an admissible test function for $\boldsymbol{R e s}_{\mathbf{2}}$, the jump contribution

$$
\mathbf{J}_{\mathbf{2}}\left(\mathbf{u}_{h}, \mathbf{v}_{h}\right)=0
$$

vanishes. A comparison with (4.2) shows, for $\mathbf{v}_{h} \in \mathbf{N d}_{\mathbf{1}}\left(\Omega ; \mathscr{T}_{h}\right)$, that

$\operatorname{Res}_{\mathbf{2}}\left(\mathbf{v}_{h}\right)=\mathbf{c}\left(\mathbf{u}_{h}-\tilde{\mathbf{u}}_{h}, \mathbf{v}_{h}\right)+\left(\mu^{-1} \operatorname{curl}_{h}\left(\mathbf{u}_{h}-\tilde{\mathbf{u}}_{h}\right), \operatorname{curl}_{h} \mathbf{v}_{h}\right)_{0, \Omega}-\mathbf{J}_{\mathbf{1}}\left(\mathbf{u}_{h}, \mu^{-1} \operatorname{curl}_{h} \mathbf{v}_{h}\right)$.

Since $\operatorname{curl}_{h} \operatorname{curl}_{h} \mathbf{v}_{h}=0$ and $\left[\gamma_{t}\left(\tilde{\mathbf{u}}_{h}\right)\right]=0$, Stokes theorem yields

$$
\begin{aligned}
& \left(\mu^{-1} \operatorname{curl}_{h}\left(\mathbf{u}_{h}-\tilde{\mathbf{u}}_{h}\right), \operatorname{curl}_{h} \mathbf{v}_{h}\right)_{0, \Omega}=\sum_{T \in \mathscr{T}_{h}} \int_{T} \mu^{-1} \operatorname{curl}_{h}\left(\mathbf{u}_{h}-\tilde{\mathbf{u}}_{h}\right) \cdot \operatorname{curl}_{h} \mathbf{v}_{h} d x= \\
& =\sum_{F \in \mathscr{F}_{h}(\Omega)} \pi_{t}\left(\mu^{-1} \operatorname{curl}_{h} \mathbf{v}_{h}\right) \cdot\left[\gamma_{t}\left(\mathbf{u}_{h}\right)\right] d \sigma=\mathbf{J}_{\mathbf{1}}\left(\mathbf{u}_{h}, \mu^{-1} \operatorname{curl}_{h} \mathbf{v}_{h}\right) .
\end{aligned}
$$

This implies the assertion of the lemma.

The unified theory leads to the following result which is stronger that the estimate of [34]. In fact, it implies the estimate [34] if one employs $\xi \lesssim \bar{\xi}$. 
Proposition 4.1. With volume and face contributions for some new

$$
\eta^{2}:=\sum_{T \in \mathscr{T}_{h}} \eta_{T}^{2}+\sum_{F \in \mathscr{F}_{h}(\Omega)} \eta_{F}^{2}
$$

defined, for $T \in \mathscr{T}_{h}$ and $F \in \mathscr{F}_{h}(\Omega)$, by

$$
\begin{aligned}
& \eta_{T}:=h_{T}\left\|\mathbf{f}-\sigma \mathbf{u}_{h}-\operatorname{curl}_{h} \mu^{-1} \operatorname{curl}_{h} \mathbf{u}_{h}\right\|_{0, T}+h_{T}\left\|\operatorname{div}\left(\mathbf{f}-\sigma \mathbf{u}_{h}\right)\right\|_{0, T}, \\
& \eta_{F}:=h_{F}^{1 / 2}\left\|\left[\pi_{t}\left(\mu^{-1} \operatorname{curl}_{h}\right) \mathbf{u}_{h}\right]\right\|_{0, F}+h_{F}^{1 / 2}\left\|\mathbf{n}_{F} \cdot\left[\sigma \mathbf{u}_{h}\right]\right\|_{0, F}
\end{aligned}
$$

it holds that

$$
\|\left(\mathbf{u}-\tilde{\mathbf{u}}_{h}, \mathbf{p}-\tilde{\mathbf{p}}_{h}\left\|_{\mathbf{V} \times \mathbf{Q}} \approx\right\| \operatorname{Res}_{\mathbf{1}}\left\|_{\mathbf{Q}^{*}}+\right\| \operatorname{Res}_{\mathbf{2}} \|_{\mathbf{V}^{*}} \lesssim \eta+\xi .\right.
$$

Proof. Lemma 4.1 suggests to consider the new functional

$$
\operatorname{Res}_{3}:=\operatorname{Res}_{2}-\mathbf{c}\left(\mathbf{u}_{h}-\tilde{\mathbf{u}}_{h}, \cdot\right)=\ell_{2}-\mathbf{b}\left(\cdot, \mu^{-1} \operatorname{curl} \tilde{\mathbf{u}}_{h}\right)-\mathbf{c}\left(\mathbf{u}_{h}, \cdot\right),
$$

which is the form of the functional $\mathbf{R e s}_{\mathbf{2}}$ in Proposition 3.2 and indeed satisfies

$$
\operatorname{Nd}_{\mathbf{1}}\left(\Omega ; \mathscr{T}_{h}\right) \subset \operatorname{Ker}\left(\operatorname{Res}_{3}\right) .
$$

This is (3.11) when $\boldsymbol{R e s}_{\mathbf{2}}$ there is replaced by $\mathbf{R e s}_{\mathbf{3}}$ from this proof. Consequently, with the new estimators defined in the proposition,

$$
\left\|\operatorname{Res}_{\mathbf{3}}\right\|_{\mathbf{V}^{*}}^{2} \lesssim \eta^{2}:=\sum_{T \in \mathscr{T}_{h}} \eta_{T}^{2}+\sum_{F \in \mathscr{F}_{h}(\Omega)} \eta_{F}^{2} .
$$

We thus obtain

$$
\left\|\operatorname{Res}_{\mathbf{2}}\right\|_{\mathbf{V}^{*}} \lesssim \eta+\left\|\mathbf{u}_{h}-\tilde{\mathbf{u}}_{h}\right\|_{0, \Omega} \leqslant \eta+\xi
$$

which concludes the proof.

\section{MORTAR EDGE ELEMENT APPROXIMATIONS}

We consider the so-called macrohybrid formulation of (3.1) in case $\mathbf{f} \in \mathbf{H}_{0}(\operatorname{div} ; \Omega)$ with respect to a non overlapping decomposition of the computational domain $\Omega$ into $N$ mutually disjoint subdomains

$$
\bar{\Omega}=\bigcup_{j=1}^{N} \bar{\Omega}_{j} \quad \text { with } \quad \Omega_{j} \cap \Omega_{k} \neq \emptyset \quad \text { for all } 1 \leqslant j<k \leqslant N .
$$

We assume the decomposition to be geometrically conforming, i.e., two adjacent subdomains either share a face, an edge, or a vertex. The skeleton $S$ of the decomposition

$$
S=\bigcup_{m=1}^{M} \bar{\gamma}_{m}
$$


consists of the interfaces $\gamma_{1}, \ldots, \gamma_{M}$ between all adjacent subdomains $\Omega_{j}$ and $\Omega_{k}$. We refer to $\gamma_{m(j)}$ as the mortar associated with subdomain $\Omega_{j}$, while the other face, which geometrically occupies the same place, is denoted by $\delta_{m(j)}$ and is called the nonmortar. Based on (5.1) we introduce the product space

$$
\mathbf{X}:=\left\{\mathbf{u} \in \mathbf{L}^{2}(\Omega)|\forall j=1, \ldots, N, \mathbf{u}|_{\Omega_{j}} \in \mathbf{H}\left(\operatorname{curl} ; \Omega_{j}\right) \text { and }\left.\gamma_{\mathbf{t}}(\mathbf{u})\right|_{\partial \Omega_{j} \cap \partial \Omega}=0\right\}
$$

equipped with the norm

$$
\|\mathbf{u}\|_{\mathbf{X}}:=\left(\sum_{j=1}^{N}\|\mathbf{u}\|_{\mathbf{c u r l}, \Omega_{j}^{2}}\right)^{1 / 2}
$$

A subdomainwise application of Stokes' theorem shows that vanishing jumps

$$
\gamma_{\mathbf{t}}(\mathbf{u})_{\gamma_{m}}=0 \text { for all } 1 \leqslant m \leqslant M
$$

of some $\mathbf{u} \in \mathbf{X}$ imply

$$
\mathbf{u} \in \mathbf{V}:=\mathbf{H}_{\mathbf{0}}(\operatorname{curl} ; \Omega) .
$$

In general, we cannot expect (5.5) to hold true and need to enforce weak continuity of the tangential traces across $\gamma_{m}$ by means of Lagrange multipliers in the space

$$
\mathbf{M}(S):=\prod_{m=1}^{M} \mathbf{H}^{-1 / 2}\left(\operatorname{div}_{\tau} ; \gamma_{m}\right)
$$

equipped with the norm

$$
\|\mu\|_{\mathbf{M}(S)}:=\left(\sum_{m=1}^{M}\left\|\left.\mu\right|_{\gamma_{m}}\right\|_{-1 / 2, \operatorname{div}_{\tau}, \gamma_{m}}\right)^{1 / 2}
$$

We introduce the bilinear form $\mathbf{A}(\cdot, \cdot): \mathbf{X} \times \mathbf{X} \rightarrow \mathbb{R}$ as the sum of the bilinear forms associated with the subdomain problems according to

$$
\mathbf{A}(\mathbf{u}, \mathbf{v}):=\sum_{j=1}^{N} a_{\Omega_{j}}\left(\left.\mathbf{u}\right|_{\Omega_{j}},\left.\mathbf{v}\right|_{\Omega_{j}}\right)=\sum_{j=1}^{N} \int_{\Omega_{j}}\left(\mu^{-1} \operatorname{curlu} \cdot \operatorname{curl} \mathbf{v}+\sigma \mathbf{u} \cdot \mathbf{v}\right) d x .
$$

Furthermore, we define the bilinear form $\mathbf{B}(\cdot, \cdot): \mathbf{X} \times \mathbf{M}(S) \rightarrow \mathbb{R}$ by means of

$$
\mathbf{B}(\mathbf{u}, \mu):=<\mu,\left[\gamma_{\mathbf{t}}(\mathbf{u})\right]>_{-1 / 2, S}
$$

with the abbreviation

$$
<\cdot,>_{-1 / 2, S}:=\sum_{m=1}^{M}<\cdot, \cdot>_{-1 / 2, \gamma_{m}} .
$$


The macro-hybrid variational formulation of (3.1a),(3.1b) reads: Find $(\mathbf{u}, \lambda) \in \mathbf{X} \times$ $\mathbf{M}(S)$ such that

$$
\begin{aligned}
& \mathbf{A}(\mathbf{u}, \mathbf{v})+\mathbf{B}(\mathbf{u}, \lambda)=\ell(\mathbf{v}) \quad \text { for all } \mathbf{v} \in \mathbf{X} \\
& \mathbf{B}(\mathbf{u}, \mu) \quad=0 \quad \text { for all } \mu \in \mathbf{M}(S) \text {. }
\end{aligned}
$$

The bilinear form $\mathbf{A}(\cdot, \cdot)$ is elliptic on the kernel of the operator associated with the bilinear form $\mathbf{B}(\cdot, \cdot)$ and $\mathbf{B}(\cdot, \cdot)$ satisfies the inf-sup condition

$$
0<\beta \leqslant \inf _{\mu \in \mathbf{M}(S)} \sup _{\mathbf{v} \in \mathbf{X}} \frac{\mathbf{B}(\mathbf{v}, \mu)}{\|\mathbf{v}\|_{\mathbf{X}}\|\mu\|_{\mathbf{M}(S)}} .
$$

The macro-hybrid variational formulation (5.11) has a unique solution $(\mathbf{u}, \lambda)$.

The mortar edge element approximation of (3.2) mimics the macro-hybrid formulation (5.11) in the discrete regime and is based on individual shape-regular simplicial triangulations $\mathscr{T}_{1}, \ldots, \mathscr{T}_{N}$ of the subdomains $\Omega_{1}, \ldots, \Omega_{N}$ regardless the situation on the skeleton $S$ of the decomposition. In particular, the interfaces inherit two different non-matching triangulations. The discretization of

$$
\mathbf{H}_{\mathbf{0}, \partial \Omega_{i} \cap \partial \Omega}\left(\operatorname{curl} ; \Omega_{j}\right):=\left\{\mathbf{u} \in \mathbf{H}\left(\operatorname{curl} ; \Omega_{j}\right) \mid \gamma_{\mathbf{t}}(\mathbf{u}) \partial \Omega_{j} \cap \partial \Omega=0\right\}
$$

with curl-conforming edge elements of Nédélec's first family [36] considers the edge element spaces $\mathbf{N d}_{\mathbf{1}, \Gamma}\left(\Omega_{j} ; \mathscr{T}_{j}\right)$ of vector fields with vanishing tangential trace on $\Gamma \cap \partial \Omega_{j}$. For a triangle $T \in \mathscr{T}_{\delta_{(k)}}$ of diameter $h_{T}$ with the surface $\delta_{m(k)} \subset S$, let $\mathbf{R T}_{\mathbf{0}}(T)$ be the lowest order Raviart-Thomas element (cf., e.g., [15]). We denote by $\mathbf{R} \mathbf{T}_{\mathbf{0}}\left(\delta_{m(k)} ; \mathscr{T}_{\delta_{m(k)}}\right)$ the associated mixed finite element space, and we refer to $\mathbf{R T}_{\mathbf{0 , 0}}\left(\delta_{m(k)} ; \mathscr{T}_{\left.\delta_{(k)}\right)}\right)$ as the subspace of vector fields with vanishing normal components on $\delta_{m(k)}$. Based on these definitions, the product space

$$
\mathbf{X}_{h}:=\left\{\mathbf{v}_{h} \in \mathbf{L}^{\mathbf{2}}(\Omega)\left|\forall j=1, \ldots, N, \mathbf{v}_{h}\right|_{\Omega_{j}} \in \mathbf{N d}_{\mathbf{1}, \Gamma}\left(\Omega_{j} ; \mathscr{T}_{j}\right)\right\}
$$

is equipped with the norm

$$
\left\|\mathbf{v}_{h}\right\| \mathbf{X}_{h}:=\left(\left\|\mathbf{v}_{h}\right\|_{\mathbf{X}}^{2}+\left\|\left.\left[\gamma_{\mathbf{t}}\left(\mathbf{v}_{h}\right)\right]\right|_{S}\right\|_{+1 / 2, h, S}^{2}\right)^{1 / 2} \text { for all } \mathbf{v}_{h} \in \mathbf{X}_{h} ;
$$

where $\|\cdot\|_{+1 / 2, h, S}$ is given by

$$
\left\|\left.\left[\gamma_{\mathbf{t}}\left(\mathbf{v}_{h}\right)\right]\right|_{S}\right\|_{+1 / 2, h, S}:=\left(\sum_{m=1}^{M}\left\|\left.\left[\gamma_{\mathbf{t}}\left(\mathbf{v}_{h}\right)\right]\right|_{\gamma_{m}}\right\|_{+1 / 2, h, \gamma_{m}}\right)^{1 / 2}
$$

and $\|\cdot\|_{+1 / 2, h, \gamma_{m}}$ stands for the mesh-dependent norm

$$
\left\|\left.\left[\gamma_{\mathbf{t}}\left(\mathbf{v}_{h}\right)\right]\right|_{\gamma_{m}}\right\|_{+1 / 2, h, \gamma_{m}}:=h^{-1 / 2}\left\|\left.\left[\gamma_{\mathbf{t}}\left(\mathbf{v}_{h}\right)\right]\right|_{\gamma_{m}}\right\|_{0, \gamma_{m}} .
$$


Due to the occurrence of nonconforming edges on the interfaces between adjacent subdomains, there is a lack of continuity across the interfaces: neither the tangential traces $\gamma_{\mathbf{t}}\left(\mathbf{v}_{h}\right)$ nor the tangential trace components $\pi_{\mathbf{t}}\left(\mathbf{v}_{h}\right)$ can be expected to be continuous. We note that $\left.\gamma_{\mathbf{t}}\left(\mathbf{v}_{h}\right)\right|_{\delta_{m(j)}} \in \mathbf{R T}_{\mathbf{0}}\left(\delta_{m(j)} ; \mathscr{T}_{\delta_{m(j)}}\right)$ and $\left.\pi_{\mathbf{t}}\left(\mathbf{v}_{h}\right)\right|_{\delta_{m(j)}} \in$ $\mathbf{N d}_{\mathbf{1}}\left(\delta_{m(j)} ; \mathscr{T}_{\left.\delta_{m(j)}\right)}\right)$. Therefore, continuity can be enforced either in terms of the tangential traces or the tangential trace components. If we choose the tangential traces, the multiplier space $\mathbf{M}_{h}(S)$ can be constructed according to

$$
\mathbf{M}_{h}(S):=\prod_{m=1}^{M} \mathbf{M}_{h}\left(\boldsymbol{\delta}_{m(j)}\right)
$$

with $\mathbf{M}_{h}\left(\boldsymbol{\delta}_{m(j)}\right)$ chosen such that

$$
\begin{aligned}
& \mathbf{R T}_{\mathbf{0 , 0}}\left(\boldsymbol{\delta}_{m(j)} ; \mathscr{T}_{\delta_{m(j)}}\right) \subset \mathbf{M}_{h}\left(\boldsymbol{\delta}_{m(j)}\right), \\
& \operatorname{dim} \mathbf{M}_{h}\left(\boldsymbol{\delta}_{m(j)}\right)=\operatorname{dim} \mathbf{R T}_{\mathbf{0 , 0}}\left(\boldsymbol{\delta}_{m(j)} ; \boldsymbol{\delta}_{m(j)}\right) .
\end{aligned}
$$

We refer to [48] for the explicit construction. The multiplier space $\mathbf{M}_{h}(S)$ will be equipped with the mesh-dependent norm

$$
\left\|\mu_{h}\right\|_{\mathbf{M}_{h}(S)}:=\left(\sum_{m=1}^{M}\left\|\left.\mu_{h}\right|_{\delta_{m(j)}}\right\|_{-1 / 2, h, \delta_{m(j)}}\right)^{1 / 2}
$$

where

$$
\left\|\left.\mu_{h}\right|_{\delta_{m(j)}}\left|\left\|_{-1 / 2, h, \delta_{m(j)}}:=h^{1 / 2}\right\| \mu_{h}\right|_{\delta_{m(j)}}\right\|_{0, \delta_{m(j)}} .
$$

The mortar edge element approximation of (3.1a),(3.1b) then requires the solution of the saddle point problem: Find $\left(\mathbf{u}_{h}, \lambda_{h}\right) \in \mathbf{X}_{h} \times \mathbf{M}_{h}(S)$ such that

$$
\begin{aligned}
\mathbf{A}_{h}\left(\mathbf{u}_{h}, \mathbf{v}_{h}\right)+\mathbf{B}_{h}\left(\mathbf{v}_{h}, \lambda_{h}\right) & =\ell\left(\mathbf{v}_{h}\right) \text { for } \quad \mathbf{v}_{h} \in \mathbf{X}_{h}, \\
\mathbf{B}_{h}\left(\mathbf{u}_{h}, \mu_{h}\right) & =0 \text { for } \mu_{h} \in \mathbf{M}_{h}(S),
\end{aligned}
$$

where the bilinear forms $\mathbf{A}_{h}(\cdot, \cdot): \mathbf{X}_{h} \times \mathbf{X}_{h} \rightarrow \mathbb{R}$ and $\mathbf{B}_{h}(\cdot, \cdot): \mathbf{X}_{h} \times \mathbf{M}_{h}(S) \rightarrow \mathbb{R}$ are given by the restriction of $\mathbf{A}(\cdot, \cdot)$ and $\mathbf{B}(\cdot, \cdot)$ to $\mathbf{X}_{h} \times \mathbf{X}_{h}$ and $\mathbf{X}_{h} \times \mathbf{M}_{h}(S)$, respectively.

Proposition 5.1. The mortar edge element approximation (5.21) admits a unique solution $\left(\mathbf{u}_{h}, \lambda_{h}\right) \in \mathbf{X}_{h} \times \mathbf{M}_{h}(S)$.

Proof. As has been shown in [48], the bilinear form $\mathbf{A}_{h}(\cdot, \cdot)$ is elliptic on the kernel of the operator associated with the bilinear form $\mathbf{B}_{h}(\cdot, \cdot)$ and that $\mathbf{B}_{h}(\cdot, \cdot)$ satisfies the inf-sup condition

$$
0<\beta \leqslant \inf _{\mu_{h} \in \mathbf{M}_{h}(S)} \sup _{\mathbf{v}_{h} \in \mathbf{X}_{h}} \frac{\mathbf{B}_{h}\left(\mathbf{v}_{h}, \mu_{h}\right)}{\left\|\mathbf{v}_{h}\right\| \mathbf{X}_{h}\left\|\mu_{h}\right\|_{\mathbf{M}_{h}(S)}} .
$$

This concludes the proof. 
In the framework of Section 3, with the minimizer $\tilde{\mathbf{u}}_{h} \in \mathbf{V}$ of the consistency error $\xi$ as given by (4.3) and $\tilde{\mathbf{p}}_{h}:=\mu^{-1}$ curl $\tilde{\mathbf{u}}_{h}$ we find

$$
\left\|\left(\mathbf{u}-\tilde{\mathbf{u}}_{h}, \mathbf{p}-\tilde{\mathbf{p}}_{h}\right)\right\|_{\mathbf{V} \times \mathbf{Q}} \approx\left\|\operatorname{Res}_{\mathbf{2}}\right\|_{\mathbf{V}^{*}},
$$

where

$$
\begin{aligned}
\operatorname{Res}_{\mathbf{2}}(\mathbf{v}) & =\sum_{i=1}^{N} \operatorname{Res}_{\mathbf{2}}^{(\mathbf{i})}(\mathbf{v}) \\
\operatorname{Res}_{\mathbf{2}}^{(\mathbf{i})}(\mathbf{v}) & :=(\mathbf{f}, \mathbf{v})_{0, \Omega_{i}}-\left(\mu^{-1} \operatorname{curl} \tilde{\mathbf{u}}_{h}, \operatorname{curl} \mathbf{v}\right)_{0, \Omega_{i}}-\left(\sigma \tilde{\mathbf{u}}_{h}, \mathbf{v}\right)_{0, \Omega_{i}} .
\end{aligned}
$$

Denoting by $\mathbf{N d}_{\mathbf{1}, 0}\left(\Omega_{i} ; \mathscr{T}_{h_{i}}\right)$ the subspace of $\mathbf{N d}_{\mathbf{1}}\left(\Omega_{i} ; \mathscr{T}_{h_{i}}\right)$ with vanishing tangential trace on $\partial \Omega_{i}$, a comparison with (5.21) shows that, for $\mathbf{v}_{h} \in \prod_{i=1}^{N} \mathbf{N d}_{\mathbf{1 , 0}}\left(\Omega_{i} ; \mathscr{T}_{h_{i}}\right)$, it holds

$$
\begin{aligned}
\operatorname{Res}_{2}\left(\mathbf{v}_{h}\right) & =\sum_{i=1}^{N} \operatorname{Res}_{\mathbf{2}}^{(\mathbf{i})}\left(\mathbf{v}_{h}\right) \\
\operatorname{Res}_{\mathbf{2}}^{(\mathbf{i})}\left(\mathbf{v}_{h}\right) & :=\left(\sigma\left(\mathbf{u}_{h}-\tilde{\mathbf{u}}_{h}, \mathbf{v}_{h}\right)_{0, \Omega_{i}}+\left(\mu^{-1} \operatorname{curl}_{h}\left(\mathbf{u}_{h}-\tilde{\mathbf{u}}_{h}\right), \operatorname{curl} \mathbf{v}_{h}\right)_{0, \Omega_{i}} .\right.
\end{aligned}
$$

Proposition 5.2. Let $\eta$ consist of element residuals $\eta_{T}$ and face residuals $\eta_{F}$ according to

$$
\eta^{2}:=\sum_{i=1}^{N}\left(\sum_{T \in \mathscr{T}_{i}} \eta_{T}^{2}+\sum_{F \in \mathscr{F}_{h}\left(\Omega_{i}\right)} \eta_{F}^{2}\right)
$$

where $\eta_{T}$ and $\eta_{F}$ are given by

$$
\begin{aligned}
& \eta_{T}:=h_{T}\left\|\mathbf{f}-\operatorname{curl} \mu^{-1} \operatorname{curl}_{h}-\sigma \mathbf{u}_{h}\right\|_{0, T}+h_{T}\left\|\operatorname{div}\left(\sigma \mathbf{u}_{h}\right)\right\|_{0, T}, \\
& \eta_{F}:=h_{F}^{1 / 2}\left\|\left[\pi_{t}\left(\mathbf{p}_{h}\right)\right]\right\|_{0, F}+h_{F}^{1 / 2}\left\|\mathbf{n}_{F} \cdot\left[\sigma \mathbf{u}_{h}\right]\right\|_{0, F} .
\end{aligned}
$$

Then, it holds

$$
\left\|\left(\mathbf{u}-\tilde{\mathbf{u}}_{h}, \mathbf{p}-\tilde{\mathbf{p}}_{h}\right)\right\|_{\mathbf{V} \times \mathbf{Q}} \lesssim \eta+\xi .
$$

Proof. In view of (5.24) we define

$$
\begin{aligned}
\operatorname{Res}_{\mathbf{3}} & :=\sum_{i=1}^{N} \operatorname{Res}_{\mathbf{3}}^{(\mathbf{i})}, \\
\boldsymbol{\operatorname { R e s }}_{\mathbf{3}}^{(\mathbf{i})}: & =\operatorname{Res}_{\mathbf{2}}^{(\mathbf{i})}-\left(\left(\sigma\left(\mathbf{u}_{h}-\tilde{\mathbf{u}}_{h}, \cdot\right)_{0, \Omega_{i}}+\left(\mu^{-1}\left(\operatorname{curl}_{h}\left(\mathbf{u}_{h}-\tilde{\mathbf{u}}_{h}\right), \operatorname{curl} \cdot\right)_{0, \Omega_{i}}\right) .\right.\right.
\end{aligned}
$$


Since $\mathbf{N d}_{\mathbf{1 , 0}}\left(\Omega_{i} ; \mathscr{T}_{h_{i}}\right) \subset \operatorname{Ker} \operatorname{Res}_{\mathbf{3}}^{(\mathbf{i})}$, a subdomainwise application of Proposition 3.2 yields

$$
\left\|\operatorname{Res}_{\mathbf{3}}\right\|_{\mathbf{V}^{*}} \lesssim \eta
$$

Hence, it follows that

$$
\left\|\operatorname{Res}_{\mathbf{2}}\right\|_{\mathbf{V}^{*}} \lesssim \eta+\left\|\mathbf{u}_{h}-\tilde{\mathbf{u}}_{h}\right\|_{0, \Omega}+\left\|\operatorname{curl}_{h} \mathbf{u}_{h}-\operatorname{curl} \tilde{\mathbf{u}}_{h}\right\|_{0, \Omega}=\eta+\xi .
$$

An upper bound $\bar{\xi}$ for the consistency error $\xi$ can be derived using the techniques from [31]. In particular, we obtain

$$
\bar{\xi}^{2}:=\sum_{j=1}^{N} \sum_{F \in \mathscr{F}_{h}\left(\delta_{m(j)}\right)}\left(\eta_{F}^{2}+\hat{\eta}_{F}^{2}\right)
$$

with additional face residuals

$$
\hat{\eta}_{F}:=h_{F}^{1 / 2}\left\|\lambda_{h}-\left\{\pi_{t}\left(\mathbf{p}_{h}\right)\right\}\right\|_{0, F}+h_{F}^{1 / 2}\left\|\lambda_{h}-\left\{\mathbf{n}_{F} \cdot \sigma \mathbf{u}_{h}\right\}\right\|_{0, F}+h_{F}^{-1 / 2}\left\|\left[\gamma_{t}\left(\mathbf{u}_{h}\right)\right]\right\|_{0, F} .
$$

Here, $\lambda_{h} \in H^{-1 / 2}\left(\gamma_{m}\right)$ satisfies

$$
\left\langle\lambda_{h}, \operatorname{curl}_{\tau} \varphi\right\rangle_{-1 / 2, \gamma_{m}}=-\left\langle\lambda_{h}, \varphi\right\rangle_{-1 / 2, \gamma_{m}} \text { for all } \varphi \in H^{1 / 2}\left(\gamma_{m}\right) .
$$

\section{REFERENCES}

1. M. Ainsworth and A. Oden, A Posteriori Error Estimation in Finite Element Analysis. Wiley, Chichester, 2000.

2. A. Alonso and A. Valli, Some remarks on the characterization of the space of tangential traces of $H(r o t ; \Omega)$ and the construction of an extension operator. Manuscr. Math. (1996) 89, 159-178.

3. C. Amrouche, C. Bernardi, M. Dauge, and V. Girault, Vector potentials in three-dimensional non-smooth domains. Math. Meth. Appl. Sci. (1998) 21, 823-864.

4. D. Arnold, R. Falk, and R. Winther, Multigrid in H(div) and H(curl). Numer. Math. (2000) 85, 197-218.

5. I. Babuska and T. Strouboulis, The Finite Element Method and its Reliability. Clarendon Press, Oxford, 2001.

6. W. Bangerth and R. Rannacher, Adaptive Finite Element Methods for Differential Equations. Lectures in Mathematics. ETH-Zürich. Birkhäuser, Basel, 2003.

7. R. Beck, P. Deuflhard, R. Hiptmair, R.H.W. Hoppe,, and B. Wohlmuth, Adaptive multilevel methods for edge element discretizations of Maxwell's equations. Surveys of Math. in Industry (1999) 8, 271-312.

8. R. Beck, R. Hiptmair, R.H.W. Hoppe, and B. Wohlmuth, Residual based a posteriori error estimators for eddy current computation. $\mathrm{M}^{2}$ AN Math. Modeling and Numer. Anal. (2000) 34, $159-182$. 
9. R. Beck, R. Hiptmair, and B. Wohlmuth, Hierarchical error estimator for eddy current computation. In: Proc. 2nd European Conf. on Advanced Numer. Meth. (ENUMATH99), Jyväskylä, Finland, July 26-30, 1999 (Neittaanmäki, P. et al.; eds.), pp. 111-120, World Scientific, Singapore, 2000.

10. F. Ben Belgacem, A. Buffa, and Y. Maday, The mortar finite element method for 3D Maxwell equations: first results. SIAM J. Numer. Anal. (2001) 39, 880-901.

11. C. Bernardi, Y. Maday, and A. Patera, Domain decomposition by the mortar element method. In: Asymptotic and Numerical Methods for Partial Differential Equations with Critical Parameters (eds.: H.Kaper et al.), pp. 269-286, Reidel, Dordrecht, 1993.

12. C. Bernardi, Y. Maday, and A. Patera, A new nonconforming approach to domain decomposition: The mortar element method. In: Nonlinear partial differential equations and their applications. (eds.:H. Brezis et al.), pp. 13-51, Paris, 1994.

13. A. Bossavit, Computational Electromagnetism. Variational Formulation, Complementarity, Edge Elements. Academic Press, San Diego, 1998.

14. S. Brenner, F. Li, and L.-Y. Sung, A locally divergence.free nonconforming finite element mewthod for the reduced time-harmonic Maxwell equations. Preprint, Department of Mathematics, Louisiana State University, Baton Rouge, LA, 2007.

15. F. Brezzi and M. Fortin, Mixed and hybrid finite element methods. Springer, Berlin-HeidelbergNew York, 1991.

16. A. Buffa and Ph. Ciarlet, Jr., On traces for functional spaces realted to Maxwell's equations. Part I: An integration by parts formula in Lipschitz polyhedra. Math. Meth. Appl. Sci (2001) 24, 9-30.

17. A. Buffa and Ph. Ciarlet, Jr., On traces for functional spaces realted to Maxwell's equations. Part II: Hodge decompositions on the boundary of Lipschitz polyhedra and applications. Math. Meth. Appl. Sci (2001) 24, 31-48.

18. A. Buffa, M. Costabel, and D. Sheen, On traces for $H(c u r l, \Omega)$ in Lipschitz domains. J. Math. Anal. Appl. (2002) 276, 845-867.

19. A. Buffa, Y. Maday, and F. Rapetti, A sliding mesh-mortar method for a two-dimensional eddy currents model of electric engines. Math. Model. Numer. Anal. (2001) 35, 191-228.

20. C. Carstensen, A unifying theory of a posteriori finite element error control. Numer. Math. (2005) 100, 617-637.

21. C. Carstensen, T. Gudi, and M. Jensen, A unifying theory of a posteriori error control for discontinuous Galerkin FEM. Numer. Math. (2009) in print. Department of Mathematics, Humboldt University of Berlin, 2008.

22. C. Carstensen and R.H.W. Hoppe, Convergence analysis of an adaptive edge finite element method for the $2 d$ eddy current equations. J. Numer. Math. (2005) 13, 19-32.

23. C. Carstensen and J. Hu, A unifying theory of a posteriori error control for nonconforming finite element methods. Numer. Math. (2007) 107, 473-502.

24. C. Carstensen, J. Hu, and A. Orlando, Framework for the a posteriori error analysis of nonconforming finite elements. SIAM J. Numer. Anal. (2007) 45, 68-82.

25. K. Eriksson, D. Estep, P. Hansbo, and C. Johnson, Computational Differential Equations. Cambridge University Press, Cambridge, 1996.

26. V. Girault and P.A. Raviart, Finite Element Approximatiuon of the Navier-Stokes Equations. Lecture Notes in Mathematics 749, Springer, Berlin-Heidelberg-New York, 1979.

27. R. Hiptmair, Finite elements in computational electromagnetism. Acta Numerica (2002) 11, 237339. 
28. R.H.W. Hoppe, Mortar edge elements in $\mathrm{R}^{3}$. East-West J. Numer. Anal. (1999) 7, 159-173.

29. R.H.W. Hoppe, Adaptive domain decomposition techniques in electromagnetic field computation and electrothermomechanical coupling problems. In: Proc. 4th European Conference on Numerical Mathematics and Advanced Applications, Ischia, Italy, July 23-27, 2001 (F. Brezzi et al.; eds.), Springer, Berlin-Heidelberg-New York, 2002.

30. R.H.W. Hoppe, Adaptive multigrid and domain decomposition methods in the computation of electromagnetic fields. J. Comput. Appl. Math. (2004) 168, 245-254.

31. R.H.W. Hoppe, Adaptive mortar edge element methods in electromagnetic field computation. Contemporary Mathematics (2005) 383, 63-111.

32. R.H.W. Hoppe and J. Schöberl, Convergence of adaptive edge element methods for the 3D eddy currents equations. to appear in J. Comp. Math., 2009

33. P. Houston, I. Perugia, and D. Schötzau, Mixed discontinuous Galerkin approximation of the Maxwell operator. SIAM J. Numer. Anal. (2004) 42, 434-459.

34. P. Houston, I. Perugia, and D. Schötzau, A posteriori error estimation for discontinuous Galerkin discretizations of $H$ (curl)-elliptic partial differential equations. IMA Journal of Numerical Analysis (2007) 27, 122-150.

35. P. Monk, Finite Element Methods for Maxwell's equations. Clarendon Press, Oxford, 2003.

36. J.-C. Nédélec, Mixed finite elements in $\mathbb{R}^{3}$. Numer. Math. (1980) 35, 315-341.

37. J.-C. Nédélec, A new family of mixed finite elements in $\mathbb{R}^{3}$. Numer. Math. (1986) 50, 57-81.

38. I. Perugia, D. Schötzau, and P. Monk, Stabilized interior penalty methods for the time-harmonic Maxwell equations. Comp. Meth. Appl. Mech. Engrg. (2002) 191, 4675-4697.

39. A. Quarteroni and A. Valli, Domain Decomposition Methods for Partial Differential Equations. Clarendon Press, Oxford, 1999.

40. F. Rapetti, The mortar edge element method on non-matching grids for eddy current calculations in moving structures. Int. J. Numer. Mod. (2001) 14, 457-477.

41. F. Rapetti, A. Buffa, Y. Maday, and F. Bouillault, Simulation of a coupled magneto-mechanical system through the sliding-mesh mortar element method. COMPEL (2000) 19, 332-340.

42. F. Rapetti, Y. Maday, and F. Bouillault, Eddy current calculations in three-dimensional structures. IEEE Trans. Magnetics (2002) 38, 613-616.

43. J. Schöberl, A posteriori error estimates for Maxwell equations, Math. Comp. (2008) 77, 633649.

44. B.F. Smith, P.E. Bjørstad, and W.D. Gropp, Domain Decomposition Methods. Cambridge University Press, Cambridge, 1996.

45. A. Toselli and A. Klawonn, A FETI domain decomposition method for edge element approximations in two dimensions with discontinuous coefficients. SIAM J. Numer. Anal. (2001) 39, 932-956.

46. R. Verfürth, A Review of A Posteriori Estimation and Adaptive Mesh-Refinement Techniques. Wiley-Teubner, New York, Stuttgart, 1996.

47. B. Wohlmuth, Discretization Methods and Iterative Solvers Based on Domain Decomposition. Lecture Notes in Computational Science and Engineering, Vol. 17. Springer, Berlin-HeidelbergNew York, 2001.

48. X. Xu and R.H.W. Hoppe, On the convergence of mortar edge element methods in $\mathbb{R}^{3}$. SIAM J. Numer. Anal. (2005) 43, 1276-1294. 